\title{
Ultraviolet catastrophe of a fluctuating curved dislocation line
}

\author{
Max Boleininger, ${ }^{1, *}$ Thomas D. Swinburne $\odot,,^{2, \dagger}$ Laurent Dupuy, ${ }^{3, *}$ and Sergei L. Dudarev ${ }^{1, \S}$ \\ ${ }^{1}$ UK Atomic Energy Authority, CCFE, Culham Science Centre, Abingdon, Oxfordshire OX14 3DB, United Kingdom \\ ${ }^{2}$ Aix-Marseille Université, CNRS, CINaM UMR 7325, Campus de Luminy, F-13288 Marseille, France \\ ${ }^{3}$ DEN-Service de Recherches Métallurgiques Appliquées, CEA, Université Paris-Saclay, F-91191 Gif-sur-Yvette, France
}

(Received 11 December 2019; revised 12 March 2020; accepted 3 June 2020; published 5 August 2020)

\begin{abstract}
Plastic deformation in metals involves stress- and temperature-driven motion of dislocations, which are topological defects interacting through elastic fields. While singular and nonsingular linear elasticity theories accurately describe long-range interactions between dislocations, both exhibit the ultraviolet catastrophe in the form of negative formation energies of short-wavelength fluctuations of dislocation lines, erroneously predicting straight dislocations to be unstable. We demonstrate how the positive energy of short-wavelength line fluctuations is restored by the nonlinearity and discreteness of the dislocation core. The treatment predicts positive formation energies of dislocation line fluctuations over their entire spectrum, in quantitative agreement with atomistic simulations, and by virtue of its simplicity lends itself to a convenient implementation in dislocation dynamics.
\end{abstract}

DOI: 10.1103/PhysRevResearch.2.032033

The motion of dislocation lines, the dominant agents of plastic deformation of crystalline solids, occurs through stochastic thermal fluctuations of the line shape that propagate through the material under the effect of applied stress [1-3]. These transient fluctuations span the entire range of scales, from the very short wavelengths that characterize thermally activated motion of line edge dislocations and prismatic dislocation loops [4,5], to mesoscopic kink-mediated fluctuations of screw dislocations [6,7], up to the almost macroscopic distortions of dislocation lines in dilute alloys or irradiated materials $[8,9]$. The general occurrence of fluctuations suggests that they represent the most fundamental aspect of dislocation-mediated deformation of metals, defining the nature of brittle-ductile transitions, hardening, fracture, and creep - in other words, the entire range of phenomena that determine the lifetime of engineering structures from an aircraft engine to a nuclear reactor. Fluctuations of dislocation lines also reflect the complex nature of the transition from discrete atoms to continuous fields in the treatment of the deformation of crystalline materials, representing the foundation of mathematical analyses of microstructure and mechanical properties [10-13].

The stochastic motion of dislocations is governed by a common principle [14-18]: Perturbations in dislocation line shape are continuously introduced by the movement of the

\footnotetext{
*max.boleininger@ukaea.uk

†swinburne@cinam.univ-mrs.fr

†laurent.dupuy@cea.fr

§sergei.dudarev@ukaea.uk
}

Published by the American Physical Society under the terms of the Creative Commons Attribution 4.0 International license. Further distribution of this work must maintain attribution to the author(s) and the published article's title, journal citation, and DOI. atomic lattice, and they are opposed by a corresponding change in the free energy of the dislocation. The free-energy change effectively gives rise to line tension, which acts to straighten out thermal fluctuations in curved dislocations. An accurate representation of the entire spectrum of fluctuations is therefore requisite for any coarse-grained dislocation dynamics model that takes into account line-shape changes due to stochastic thermal effects.

The linear elasticity theory erroneously predicts that small perturbations of the dislocation line shape are energetically favorable at small wavelengths $[2,19]$, resulting in the ultraviolet catastrophe. In other words, at finite temperature a straight dislocation is predicted to be unstable with respect to short-wavelength fluctuations. While nonsingular methods have been developed to regularize the well-known divergences in strain, stress, and elastic energy close to a dislocation line [20-22], the instability of dislocations with respect to short-wavelength fluctuations persists even in these regularized models, motivating the analysis detailed below.

To understand the fundamental origin of the shortwavelength instability and develop a treatment free from these runaway ultraviolet fluctuations, we introduce a nonlinear description of the dislocation core derived from a minimal model of atomic bonding in a body-centered-cubic (bcc) crystal that accounts for periodicity in the discrete lattice $[23,24]$. By combining a linear elastic description of displacements at a large distance from a curved dislocation with the aforementioned nonlinear slip condition at the glide surface, we obtain a dislocation dynamics model that predicts a stable spectrum of shape fluctuations over the entire range of scales, with configuration energies quantitatively consistent with atomistic simulations, and containing no adjustable parameters. Furthermore, the model provides an insight into the origin of the commonly used empirical line-tension approximation for the dislocation core energy. 
The local core model. To form a dislocation we join two linear elastic domains $\Omega=\Omega^{+} \cup \Omega^{-}$separated by a glide surface $S=\partial \Omega^{+}$. Following the seminal studies by Peierls and Nabarro $[25,26]$, we introduce a nonlinear misfit potential in $S$, derived from the multistring Frenkel-Kontorova model [23,27], resulting in the Lagrangian $\mathcal{L}$ over the displacement field $u_{i}$ [24],

$$
\begin{aligned}
\mathcal{L} & =\mathcal{L}_{\Omega}+\mathcal{L}_{\partial \Omega}, \\
\mathcal{L}_{\Omega} & =-\frac{1}{2} \int_{\Omega} d V u_{i, j} \sigma_{i j}, \\
\mathcal{L}_{\partial \Omega} & =-\frac{2 \mu p b}{\pi^{2}} \int_{S} d A \sin ^{2}\left[\frac{\pi}{b} \hat{b}_{j}\left(u_{j}^{+}-u_{j}^{-}\right)\right],
\end{aligned}
$$

where $u_{i, j}=\partial_{j} u_{i}$ is the displacement field gradient, $\sigma_{i j}=$ $c_{i j k l} u_{k, l}$ is the stress tensor, $c_{i j k l}$ is the stiffness tensor, $\mu$ is the shear modulus, and $b_{i}$ is the Burgers vector. The dimensionless structure constant $p$ is obtained via a comparison to isotropic elasticity theory [24].

Tensor $u_{i, j}$ is also called the distortion tensor $\beta_{i j}$, which is related to the strain tensor $\varepsilon_{i j}=\frac{1}{2}\left(\beta_{i j}+\beta_{j i}\right)$ [28]. Fields $u_{j}^{ \pm}$are defined in terms of their limits approaching the glide surface in the direction of the surface normal $n_{j}^{ \pm}$defined in either elastic domain. The Lagrangian $\mathcal{L}_{\Omega}$ represents the elastic medium in $\Omega$ in accordance with linear elasticity theory, while $\mathcal{L}_{\partial \Omega}$ is a Peierls-Nabarro periodic energetic penalty for displacements collinear to the Burgers vector at the glide surface, reflecting the discrete lattice discontinuity at the slip plane and nonharmonicity of the interatomic interaction near the dislocation core in a bcc crystal [24].

The equilibrium equations are derived by applying the virtual work principle to the Lagrangian,

$$
\begin{aligned}
c_{i j k l} u_{k, l}^{ \pm} n_{j}^{\mp} & =\frac{2 \mu p b_{i}}{\pi} \sin \left[\frac{2 \pi}{b} \hat{b}_{j}\left(u_{j}^{+}-u_{j}^{-}\right)\right], \quad \boldsymbol{r} \in S, \\
c_{i j k l} u_{k, l j} & =0, \quad \boldsymbol{r} \in \Omega .
\end{aligned}
$$

It is sufficient to solve (2a) for the distortion field in $S$, which is then convolved with the elastic Green's function to give the solution in $\Omega$. The distortion field in $S$ thus takes the role of an inclusion, commonly referred to as the eigendistortion $\beta_{i j}^{*}$, to distinguish it from the elastic distortion $\beta_{i j}$ in $\Omega$ [29].

We exploit the linearity of the elastostatic equilibrium condition (2b) to find approximate solutions to the nonlinear boundary-value problem (2). Similar to other regularization schemes $[20,21]$, we express the eigendistortion in terms of a convolution of the singular eigendistortion of a classical dislocation by a nonsingular distribution function $\rho(\boldsymbol{r})$. To preserve the discontinuity of the displacement field across a possibly curved glide surface, the convolution must be applied in the direction perpendicular to the local normal vector to the surface; in order to obtain analytical expressions for the self-energy, we additionally choose to apply the convolution in the direction collinear to the Burgers vector, obtaining

$$
\tilde{\beta}_{i j}^{*}(\boldsymbol{r}) \simeq-b_{i} \oint_{S_{\Gamma}} d A_{j}^{\prime} \int_{-\infty}^{\infty} d s \rho\left(\boldsymbol{r}^{\prime}-s \hat{\boldsymbol{b}}\right) \delta[R(\boldsymbol{r}-s \hat{\boldsymbol{b}})],
$$

where $\delta$ is the Dirac delta function and $R$ is the Euclidean distance between point $\boldsymbol{r}$ in the bulk and $\boldsymbol{r}^{\prime}$ on the cut surface $S_{\Gamma} \subset S$. The cut surface is defined by the glide surface $S$ which is terminated by the dislocation curve $\Gamma$ lying inside the surface. The singular eigendistortion of the classical dislocation model is recovered by choosing $\rho$ as the singular Dirac delta function.

As the convolution (3) is applied before the integral over the cut surface, the distribution function ansatz $\rho(\boldsymbol{r})$ can depend on the local line direction $\hat{t}$ as well as the topologically conserved $\hat{\boldsymbol{b}}$, thus allowing a dependence on the dislocation character, parametrized by $\cos \alpha=\hat{\boldsymbol{t}} \cdot \hat{\boldsymbol{b}}$.

For a straight dislocation of arbitrary character, the eigendistortion model (3) solves the boundary-value problem (2) exactly if $\rho(\boldsymbol{r})$ is taken in the form of the CauchyLorentz distribution [24],

$$
\rho(\boldsymbol{r})=\frac{1}{\pi \kappa} \frac{\kappa^{2}}{(\boldsymbol{r} \cdot \hat{\boldsymbol{b}})^{2}+\kappa^{2}},
$$

with a dislocation core width of

$$
\kappa|\sin \alpha|=\frac{b}{8 p(1-v)}\left(1-v \cos ^{2} \alpha\right),
$$

defined as the width of the distribution perpendicular to the dislocation line [30].

The glide-surface Lagrangian (1c) predicts core widths that are too narrow compared to atomistic simulations because the finite separation $h$ between the lattice planes parallel to the glide surface is neglected. In what follows, this is accounted for by a perturbative correction to the structure constant $p$, namely [24]

$$
p \rightarrow p_{h}=\frac{b p}{b+2 h p(3-2 v)} .
$$

For a general curved dislocation, the eigendistortion (3) is found using the distribution function of the straight mixed dislocation (4) using the local line character $\cos \alpha=\hat{\boldsymbol{t}} \cdot \hat{\boldsymbol{b}}$. We refer to Fig. 1 for a visual representation of the local core distribution for the example of a perturbed edge dislocation. This approximation is valid under two conditions. First, the core distribution is assumed to depend on the line shape locally, thereby neglecting the influence of other nearby dislocations on the boundary-value problem. Second, the core distribution depends on the line shape to first order, specifically through the local line character defined by Eq. (5). The curvature of the line could be accounted for as a second-order correction to the distribution, which is neglected here. While, in principle, nonlocal effects can be included by varying the distribution $\rho(\boldsymbol{r})$ across the dislocation network in order to minimize the total free energy, the analysis given below shows that the local approach adopted here already fully solves the ultraviolet catastrophe and the corresponding short-wavelength line instability problem.

By analogy to the classical dislocation theory [31], elastic fields in the local core model are expressed as line integrals by application of the isotropic elasticity Green's function, with the eigendistortion of the local core model (3) taking on the role of an inclusion [29]. The resulting expressions are analytical; see the Supplemental Material [32]. 
Perturbed atomistic configurations

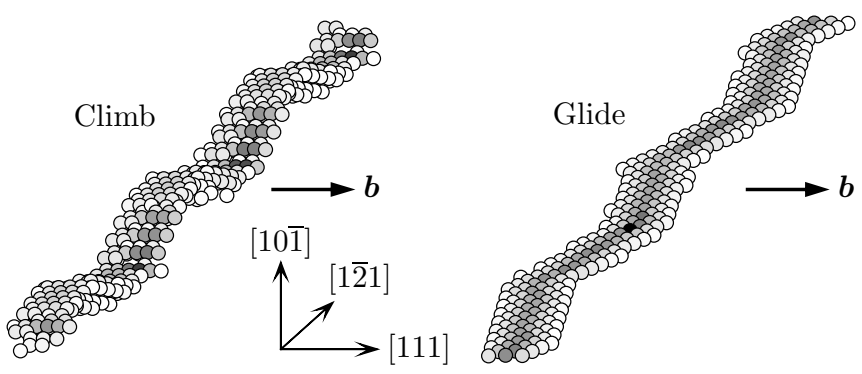

Core convolution functions
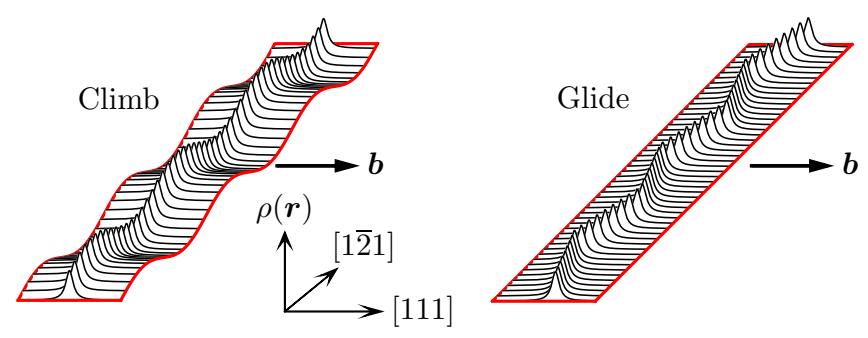

FIG. 1. Atomistic core structures of $\frac{1}{2}[111]$ edge dislocations perturbed in the climb and glide directions. The panels below show the corresponding distributions $\rho(\boldsymbol{r})$ of the eigendistortion found using the local core model, offset by the position of the glide surface.

Energies in the local core model. To apply the local core model to stochastic dislocation dynamics, we require analytical expressions for energies and forces of arbitrary dislocation configurations. Consider the total energy of two closed dislocation loops, labeled by (1) and (2), in an infinite elastic medium,

$$
W=W^{(1)}+W^{(2)}+W^{(1,2)}+W_{\text {core }}^{(1)}+W_{\text {core }}^{(2)},
$$

where $W^{(1)}$ and $W^{(2)}$ are elastic self-energies of the isolated loops and $W^{(1,2)}$ is the elastic interaction energy between the loops. The terms $W_{\text {core }}^{(1)}$ and $W_{\text {core }}^{(2)}$ represent interfacial energies (1c) in the approximation that the interfacial energy is unaffected by the presence of other dislocations. The elastic interaction energy follows from the bulk Lagrangian (1b),

$$
W^{(1,2)}=\frac{1}{2} \int_{\Omega} d V \tilde{u}_{i, j}^{(1)} \tilde{\sigma}_{i j}^{(2)},
$$

with elastic self-energies given by $W^{(1)}=\frac{1}{2} W^{(1,1)}$. Only the eigendistortion contributes to the potential energy, with the integral over elastic distortion vanishing [21]. Substituting the approximate eigendistortion (3) and stress field (see the Supplemental Material [32]) into Eq. (8), we arrive at an explicit expression for interaction energy in the local core model, namely

$$
\begin{aligned}
W^{(1,2)}= & -\frac{\mu}{8 \pi} \oint_{\Gamma^{(1)} \Gamma^{(2)}}\left\{\left[R_{, k k}\right]^{(1,2)} b_{i}^{(1)} b_{j}^{(2)} d l_{i}^{(1)} d l_{j}^{(2)}\right. \\
& +\frac{2}{1-v}\left[R_{, i j}\right]^{(1,2)} b_{i}^{(1)} b_{j}^{(2)} d l_{k}^{(1)} d l_{k}^{(2)}
\end{aligned}
$$

$$
\begin{aligned}
& -\frac{2}{1-v}\left[R_{, k k}\right]^{(1,2)} b_{i}^{(1)} b_{i}^{(2)} d l_{j}^{(1)} d l_{j}^{(2)} \\
& \left.+\frac{2 v}{1-v}\left[R_{, k k}\right]^{(1,2)} b_{i}^{(1)} b_{j}^{(2)} d l_{j}^{(1)} d l_{i}^{(2)}\right\}
\end{aligned}
$$

where

$$
\begin{aligned}
{\left[R_{, i j}\right]^{(1,2)}=} & \int_{-\infty}^{\infty} d s \int_{-\infty}^{\infty} d q \rho^{(1)}\left(q \hat{\boldsymbol{b}}^{(1)}\right) \rho^{(2)}\left(s \hat{\boldsymbol{b}}^{(2)}\right) \\
& \times \partial_{i}^{(1)} \partial_{j}^{(1)}\left\|\boldsymbol{r}^{(1)}-q \hat{\boldsymbol{b}}^{(1)}-\boldsymbol{r}^{(2)}+s \hat{\boldsymbol{b}}^{(2)}\right\|
\end{aligned}
$$

is the twice-convolved tensor of second derivatives of $R$. The formula is equivalent to De Wit's expression for the interaction energy of singular dislocation theory [33], except for the occurrence of a convolved tensor of second derivatives $\left[R_{, i j}\right]^{(1,2)}$.

For the treatment of self-interaction, where $\hat{\boldsymbol{b}}^{(1)}=\hat{\boldsymbol{b}}^{(2)}=\hat{\boldsymbol{b}}$ it is possible to express $\left[R_{, i j}\right]^{(1,2)}$ analytically in terms of the twice-convolved inverse distance $R_{c}^{-1}$,

$$
R_{c}^{-1}=\int_{-\infty}^{\infty} d s \int_{-\infty}^{\infty} d q \frac{\rho^{(1)}(q \hat{\boldsymbol{b}}) \rho^{(2)}(s \hat{\boldsymbol{b}})}{\left\|\boldsymbol{r}^{(1)}-q \hat{\boldsymbol{b}}-\boldsymbol{r}^{(2)}+s \hat{\boldsymbol{b}}\right\|},
$$

which is solved by

$$
R_{c}^{-1}=\frac{\frac{2}{\pi} \arccos \left(\sqrt{\frac{i(\boldsymbol{d} \cdot \hat{\boldsymbol{b}})+\eta+\|\boldsymbol{d}-\hat{\boldsymbol{b}}(\boldsymbol{d} \cdot \hat{\boldsymbol{b}})\|}{2\|\boldsymbol{d}-\hat{\boldsymbol{b}}(\boldsymbol{d} \cdot \hat{\boldsymbol{b}})\|}}\right)}{\sqrt{(\boldsymbol{d}-i \eta \hat{\boldsymbol{b}}) \cdot(\boldsymbol{d}-i \eta \hat{\boldsymbol{b}})}}+\text { c.c. }
$$

Here, $\eta=\kappa^{(1)}+\kappa^{(2)}, \boldsymbol{d}=\boldsymbol{r}^{(1)}-\boldsymbol{r}^{(2)}$, and c.c. refers to the complex conjugate. Tensor $\left[R_{, i j}\right]^{(1,2)}$ is subsequently evaluated using the transformation

$$
\begin{aligned}
{\left[R_{, i j}\right]^{(1,2)}=} & \delta_{i j} R_{c}^{-1}-\hat{b}_{i} \hat{b}_{j}\left(R_{c}^{-1}+d_{l} g_{l}\right) \\
& +d_{i} g_{j}+\hat{b}_{i} \hat{b}_{l}\left(d_{j} g_{l}-g_{j} d_{l}\right),
\end{aligned}
$$

where $g_{i}=\partial_{i} R_{c}^{-1}$ is the derivative of the convolved inverse distance with respect to $d_{i}$. The effective stress needed for computing the Peach-Koehler force [20,21] requires the convolved tensor of third derivatives, which is given in the Supplemental Material [32] along with the derivation of Eq. (13). Note that the single-convolved inverse distance, as required for evaluating elastic fields in the local core model, is obtained by setting $\eta$ equal to the appropriate $\kappa^{(i)}$ in Eq. (12).

The interfacial energy is expressed as a line integral by substituting the plastic slip into Lagrangian (1c),

$$
W_{\mathrm{core}}^{(i)}=\frac{p}{p_{h}} \frac{\mu b^{2}}{4 \pi(1-v)} \oint_{\Gamma^{(i)}} d l^{(i)}\left(1-v \cos ^{2} \alpha\right),
$$

where $\alpha$ varies in accordance with the parametrization of the dislocation curve. The functional form for the interfacial energy between domains $\Omega^{+}$and $\Omega^{-}$, derived here from first principles, matches the commonly used line-tension approximation for the core energy $[19,34,35]$. With the exception of the $p / p_{h}$ prefactor, the core energy does not depend on atomistic features, but rather only on the dislocation character and elastic constants. This feature follows from the requirement that the nonlinear string interaction at the glide surface must reduce to the known linear elastic interaction in the bulk region [24]. 
Perturbation formation energy $E / \lambda(\mathrm{eV} / \AA)$

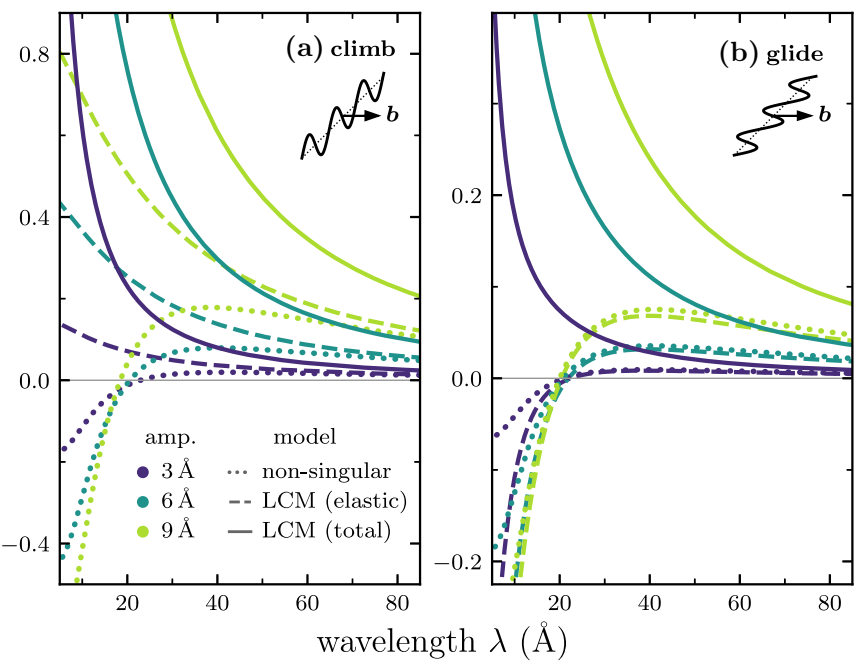

FIG. 2. Formation energy of sinusoidal perturbations in (a) climb and (b) glide directions for a $\frac{1}{2}[111](10 \overline{1})$ edge dislocation in tungsten. The nonsingular elasticity theory is compared with the local core model (LCM), with (total) and without (elastic) contribution of the core energy. The local core model is seen to be free from the small-wavelength instabilities inherent to the nonsingular theory.

Perturbations of a dislocation line. We consider periodic perturbations of an initially straight edge dislocation in either the glide or climb direction. We also compare the local core model with the nonsingular continuum theory [20]. The spreading radius $R_{C}$ of nonsingular continuum theory is chosen such that the elastic self-energy per unit length of the straight edge dislocation is identical in both models, leading to $R_{C}=2 \kappa$. Figure 2 shows a comparison of the formation energies for perturbations of sinusoidal shape of a tungsten edge dislocation, computed using the values of the material constants given in Tables I and II. The local core model eliminates the small-wavelength instability. A comparison with strain-gradient elasticity theory [21] is given in the Supplemental Material [32] with similar conclusions.

Atomistic perturbation energies were computed using LAMMPS [36] with two interatomic potentials each for bcc iron $[37,38]$ and tungsten $[39,40]$. We studied the periodic perturbations of straight edge dislocations of the types listed in Table I. Climb-perturbed dislocations were constructed by initializing dislocations with sinusoidally shifted glide surfaces, and subsequently relaxing the system. Glide-perturbed

TABLE I. Geometric and structure constants for the dislocations studied in this work. Constants $p$ and $h$ are determined in the Supplemental Material [32], and $a$ is the lattice constant.

\begin{tabular}{lccc}
\hline \hline Dislocation type & $b(a)$ & $p$ & $h(a)$ \\
\hline$\frac{1}{2}[111](10 \overline{1})$ & $\sqrt{3} / 2$ & $\sqrt{3} /(4 \sqrt{2})$ & $1 / \sqrt{2}$ \\
$\frac{1}{2}[111](1 \overline{2} 1)$ & $\sqrt{3} / 2$ & $\sqrt{3} /(4 \sqrt{2})$ & $1 / \sqrt{6}$ \\
{$[100](001)$} & 1 & $1 / \sqrt{2}$ & $1 / 2$ \\
{$[100](0 \overline{1} 1)$} & 1 & $1 /(2 \sqrt{2})$ & $1 / \sqrt{2}$ \\
\hline \hline
\end{tabular}

TABLE II. Materials constants used for continuum models.

\begin{tabular}{lccc}
\hline \hline Material & $\mu\left(\mathrm{eV} / \AA^{3}\right)$ & $v$ & $a(\AA)$ \\
\hline $\mathrm{W}$ & 1.00 & 0.28 & 3.14 \\
$\mathrm{Fe}$ & 0.39 & 0.43 & 2.84 \\
\hline \hline
\end{tabular}

dislocations were generated by first applying constraint forces to atoms such that the dislocation would bow out periodically with a specified wavelength and amplitude. Next, the constraint forces were removed, and the system evolved using Langevin dynamics at zero temperature with a damping time constant of $0.25 \mathrm{ps}$. Following a simulation time of $0.5 \mathrm{ps}$, the potential energy was found to decay exponentially over time, as is characteristic for strongly damped systems. This suggests that the energy penalty associated with the constraint forces has become negligible, enabling us to extract perturbation energy over a broad range of perturbation amplitudes.

The perturbation wavelengths were chosen as modes of the simulation box dimension in the dislocation line direction, for line lengths of 200 and $300 \AA$ in tungsten, or 150 and $220 \AA$ in iron. Atoms separated from the line farther than $300 \AA$ in tungsten, or $220 \AA$ in iron, were held fixed at positions corresponding to the anisotropic linear elasticity solution for the displacement field using elastic constants appropriate for the chosen interatomic potential. Perturbation formation energies were computed as the difference between the formation energies of the perturbed and straight configurations, only counting unfixed atoms.

The amplitudes and wavelengths of the perturbed configurations were determined by fitting a zigzag function to the positions of high-energy atoms. Data were discarded for fits with anomalously large residuals, as this indicated the loss of a regular core structure during the relaxation process. The resulting amplitudes and wavelengths were used as the input for the local core model, allowing for a direct comparison between the continuum and atomistic description. In order to resolve the significantly kinked structure of glide-perturbed [100] dislocations, we used a piecewise linear description obtained by binning the positions of high-energy atoms. We further included a Peierls energy contribution [41] using Peierls barriers extracted from atomistic nudged-elastic band [42] calculations for each interatomic potential. Details on this procedure are given in the Supplemental Material [32].

We refer to Fig. 3 for a comparison of the formation energies of line fluctuations from the local core model and atomistic simulations. Predictions by the local core model are generally consistent with the atomistic reference, showing that the continuum model can reproduce configurational energies of [111]- and [100]-type edge dislocations in bcc iron and tungsten over a broad range of line curvatures. Note that the local core model contains no adjustable or external parameters, with the exception of the Peierls barrier for dislocations of the [100] type. We find that the local core model systematically overestimates energies for climbperturbed dislocations, possibly because the effect of line curvature on the core functional is neglected. The energies for glide-perturbed dislocations are scattered, with energies for 


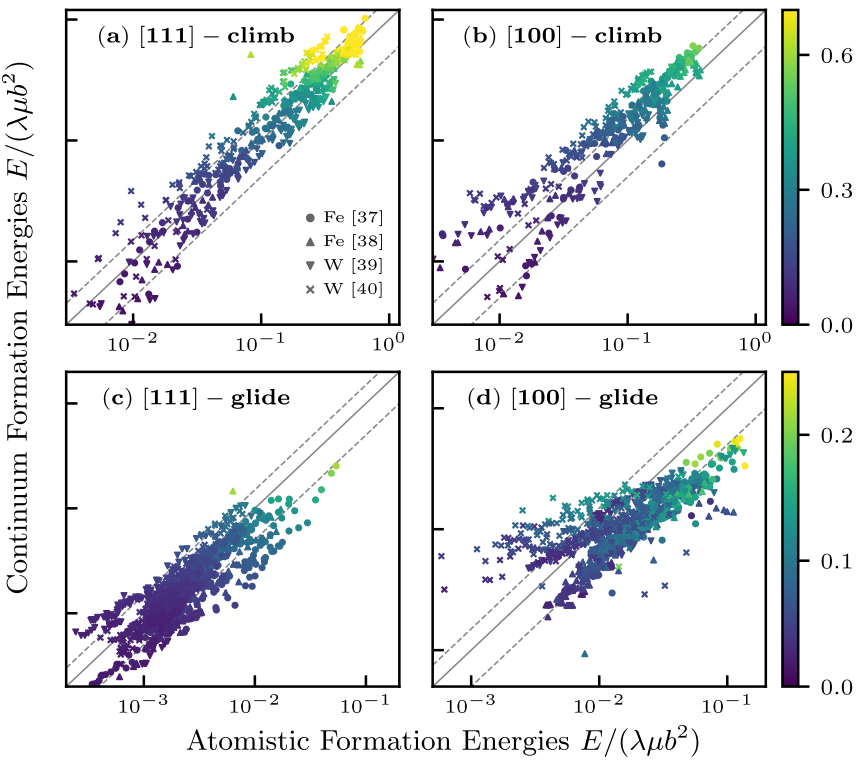

FIG. 3. Comparison of formation energies of periodic perturbations in an edge dislocation perturbed in the (a), (b) climb and (c), (d) glide directions. Atomistic energies are computed using two interatomic potentials both for iron [37,38] and tungsten [39,40]. Continuum energies are computed using the local core model for perturbed dislocations matching the corresponding atomistic configurations. Data are colored according to the ratio of perturbation amplitude to wavelength (see the color bars). Dashed lines indicate an over- or underestimation by $50 \%$.

dislocations in iron being systematically underestimated; iron is probably better described by an elastically anisotropic local core model. The chosen potentials display systematic disparities in energy for the same material, confirming that these formation energies are sensitive to the choice of the interatomic potential [43].
Averaged over all the sampled dislocations of [100] type, the Peierls energies represent a significant fraction of the total formation energies: $18 \%$ for $[100](0 \overline{1} 1)$ type, and $55 \%$ for [100](001) type. The core of a [100] dislocation is narrow, which is correlated with the large value of parameter $p$ in Table I. Consequently, the Peierls barrier is not negligible, and hence kink formation [41] contributes a significant part to the dislocation stiffness.

Concluding remarks. Based on a variational PeierlsNabarro model, we develop a dislocation dynamics formalism that takes into account the planar spreading of plastic eigendistortion across the glide surface. The local core model resolves the problem of the short-wavelength instability of dislocation lines and predicts formation energies of perturbations of the dislocation line shape qualitatively and quantitatively consistent with atomistic simulations. Furthermore, we demonstrate that the line-tension expression for the dislocation core energy and the regularization of elastic fields near the dislocation core fundamentally emerge from the periodicity of the discrete atomic lattice, here considered through a nonlinear misfit potential. By incorporating the effect of the nonlinear misfit potential into the plastic eigendistortion representing a dislocation, we arrive at a dislocation dynamics model with a physically consistent and analytically tractable description of a planar dislocation core, free from the ultraviolet catastrophe instability.

The authors gratefully acknowledge discussions with D. Rodney, P.-A. Geslin, J. B. J. Chapman, A. J. London, P.-W. Ma, and D. R. Mason. This work has been carried out within the framework of the EUROfusion Consortium and has received funding from the Euratom research and training programme 2014-2018 and 2019-2020 under Grant Agreements No. 633053 and No. 755039. Also, it has been partially funded by the RCUK Energy Programme (Grant No. $\mathrm{EP} / \mathrm{T} 012250 / 1)$. The views and opinions expressed herein do not necessarily reflect those of the European Commission.
[1] P. M. Derlet, M. R. Gilbert, and S. L. Dudarev, Phys. Rev. B 84, 134109 (2011).

[2] P. A. Geslin and D. Rodney, Phys. Rev. B 98, 174115 (2018).

[3] Y. Li, M. Boleininger, C. Robertson, L. Dupuy, and S. L. Dudarev, Phys. Rev. Materials 3, 073805 (2019).

[4] Y. N. Osetsky, D. J. Bacon, A. Serra, B. N. Singh, and S. I. Golubov, Philos. Mag. 83, 61 (2003).

[5] S. Zhao, Y. Osetsky, and Y. Zhang, Phys. Rev. Materials 3, 103602 (2019).

[6] A. Seeger, H. Donth, and F. Pfaff, Discuss. Faraday Soc. 23, 19 (1957).

[7] W. Cai, V. V. Bulatov, S. Yip, and A. S. Argon, Mater. Sci. Eng. A 309-310, 270 (2001).

[8] C. S. Deo, D. J. Srolovitz, W. Cai, and V. V. Bulatov, Phys. Rev. B 71, 014106 (2005).

[9] J. Dérès, L. Proville, and M.-C. Marinica, Acta Mater. 99, 99 (2015).

[10] L. E. Shilkrot, R. E. Miller, and W. A. Curtin, Phys. Rev. Lett. 89, 025501 (2002).
[11] J. Marian, W. Cai, and V. V. Bulatov, Nat. Mater. 3, 158 (2004).

[12] K. Kang, V. V. Bulatov, and W. Cai, Proc. Natl. Acad. Sci. USA 109, 15174 (2012).

[13] P. M. Derlet and S. L. Dudarev, Phys. Rev. Materials 4, 023605 (2020).

[14] M. Hiratani and H. M. Zbib, J. Eng. Mater. Technol. 124, 335 (2002).

[15] M. Hiratani and H. M. Zbib, J. Nucl. Mater. 323, 290 (2003).

[16] A. Stukowski, D. Cereceda, T. D. Swinburne, and J. Marian, Int. J. Plast. 65, 108 (2015).

[17] T. D. Swinburne, K. Arakawa, H. Mori, H. Yasuda, M. Isshiki, K. Mimura, M. Uchikoshi, and S. L. Dudarev, Sci. Rep. 6, 30596 (2016)

[18] S. Hayakawa, T. Okita, M. Itakura, M. Aichi, and K. Suzuki, Philos. Mag. 98, 2311 (2018).

[19] B. A. Szajewski, F. Pavia, and W. A. Curtin, Modell. Simul. Mater. Sci. Eng. 23, 085008 (2015).

[20] W. Cai, A. Arsenlis, C. R. Weinberger, and V. V. Bulatov, J. Mech. Phys. Solids 54, 561 (2006). 
[21] G. Po, M. Lazar, D. Seif, and N. Ghoniem, J. Mech. Phys. Solids 68, 161 (2014).

[22] M. Lazar, Philos. Mag. 97, 3246 (2017).

[23] M. Boleininger, T. D. Swinburne, and S. L. Dudarev, Phys. Rev. Materials 2, 083803 (2018).

[24] M. Boleininger and S. L. Dudarev, Phys. Rev. Materials 3, 093801 (2019).

[25] R. Peierls, Proc. Phys. Soc. 52, 34 (1940).

[26] F. R. N. Nabarro, Proc. Phys. Soc. 59, 256 (1947).

[27] S. L. Dudarev, Philos. Mag. 83, 3577 (2003).

[28] L. D. Landau and E. M. Lifshitz, Theory of Elasticity (Butterworth, Oxford, UK, 1986).

[29] T. Mura, Micromechanics of Defects in Solids (Springer, Berlin, 2013).

[30] Note that while $\kappa$ is infinite in the pure screw orientation, the core width in the direction perpendicular to the line remains finite in the limit approaching the pure screw orientation. The screw dislocation core is therefore regularized and planar.

[31] P. M. Anderson, J. P. Hirth, and J. Lothe, Theory of Dislocations (Cambridge University Press, Cambridge, UK, 2017).

[32] See Supplemental Material at http://link.aps.org/supplemental/ 10.1103/PhysRevResearch.2.032033 for derivations of the structure constants $p$ and $h$, the analytical expressions for elastic fields of curved dislocations in the local core model, and the convolved tensor of third derivatives, and which also includes a comparison to strain-gradient theory, and Peierls barriers of the [100] edge dislocations.

[33] R. de Wit, in Solid State Physics (Elsevier, Amsterdam, 1960), Vol. 10, pp. 249-292.

[34] A. V. Granato and K. Lücke, J. Appl. Phys. 27, 583 (1956).

[35] L. Dupuy and M. C. Fivel, Acta Mater. 50, 4873 (2002).

[36] S. Plimpton, J. Comput. Phys. 117, 1 (1995).

[37] G. J. Ackland, D. J. Bacon, A. F. Calder, and T. Harry, Philos. Mag. A 75, 713 (1997).

[38] P. A. Gordon, T. Neeraj, and M. I. Mendelev, Philos. Mag. 91, 3931 (2011).

[39] M.-C. Marinica, L. Ventelon, M. R. Gilbert, L. Proville, S. L. Dudarev, J. Marian, G. Bencteux, and F. Willaime, J. Phys.: Condens. Matter 25, 395502 (2013).

[40] D. R. Mason, D. Nguyen-Manh, and C. S. Becquart, J. Phys.: Condens. Matter 29, 505501 (2017).

[41] T. D. Swinburne, S. L. Dudarev, S. P. Fitzgerald, M. R. Gilbert, and A. P. Sutton, Phys. Rev. B 87, 064108 (2013).

[42] G. Henkelman, B. P. Uberuaga, and H. Jónsson, J. Chem. Phys. 113, 9901 (2000).

[43] D. R. Mason, D. Nguyen-Manh, M.-C. Marinica, R. Alexander, A. E. Sand, and S. L. Dudarev, J. Appl. Phys. 126, 075112 (2019). 